\title{
Radiation-induced gastrointestinal syndrome is alleviated in NDRG2-deficient mice
}

\author{
Jun Zhu ${ }^{1 \#}$, Lianlian Tian ${ }^{2 \#}$, Huichen $\mathrm{Li}^{3 \#}$, Jun $\mathrm{Hao}^{4}$, Shuai Wang ${ }^{1}$, Jipeng $\mathrm{Li}^{1}$, Jian Zhang ${ }^{3}$ \\ ${ }^{1}$ State Key Laboratory of Cancer Biology, Institute of Digestive Diseases, Xijing Hospital, The Fourth Military Medical University, Xi'an, China; \\ ${ }^{2}$ Department of Pediatrics, Tangdu Hospital, Air Force Medical University, Xi'an, China; ${ }^{3}$ State Key Laboratory of Cancer Biology, Department of \\ Biochemistry and Molecular Biology, The Fourth Military Medical University, Xi'an, China; ${ }^{4}$ Department of Experiment Surgery, Xijing Hospital, \\ Fourth Military Medical University, Xi'an, China \\ Contributions: (I) Conception and design: J Zhu; (II) Administrative support: J Li, J Zhang; (III) Provision of study materials or patients: L Tian; \\ (IV) Collection and assembly of data: H Li; (V) Data analysis and interpretation: J Hao, S Wang; (VI) Manuscript writing: All authors; (VII) Final \\ approval of manuscript: All authors. \\ "These authors contributed equally to this work. \\ Correspondence to: Jipeng Li, MD, PhD. State Key Laboratory of Cancer Biology, Institute of Digestive Diseases, Xijing Hospital, The Fourth \\ Military Medical University, Xi'an, China. Email: jipengli1974@aliyun.com; Jian Zhang, MD, PhD. Department of Biochemistry and Molecular \\ Biology, the Fourth Military Medical University, Xi’an, China. Email: biozhangj@fmmu.edu.cn.
}

Background: Radiation-induced gastrointestinal syndrome (GIS) often occurs after therapeutic or accidental exposure to high doses of radiation. Unfortunately, there are still no effective medical treatments for GIS. N-Myc downstream regulated gene 2 (NDRG2), is a tumor suppressor gene and promotes cell apoptosis and differentiation. The aim of our study was to identify the role of NDRG2 in the progression of GIS and explore the potential mechanism.

Methods: We generated Ndrg2 ${ }^{\Delta G}$ mice, lacking NDRG2 specifically in the intestinal epithelium. Survival analysis was performed to validate the effect of NDRG2 on GIS, and other common indicators (body weight loss and diarrhea) were used for the assessment of GIS. Enzyme-linked immunosorbent assay (ELISA) and reverse transcription-polymerase chain reaction (RT-PCR) were conducted to obtain the expression of proinflammatory interleukin (IL)-1 $\beta$, IL-6, and tumor necrosis factor alpha (TNF- $\alpha$ ). TUNNEL and western blotting were further adopted to determine the relationship between NDRG2 and apoptosis. Finally, we performed histology and immunohistochemistry assays to explore the morphological alternations and changes of proliferation-related molecules, including Ki-67 and proliferating cell nuclear antigen (PCNA).

Results: We found that after 8 gray of total body $r$-irradiation (TBI), the deletion of NDRG2 in the intestine revealed longer survival time, considerably milder symptoms of GIS, and milder damage to jejunal tissue, compared with the WT mice. Moreover, the $\mathrm{Ndrg} 2^{\Delta \mathrm{G}}$ mice significantly inhibited the expression of pro-inflammatory IL-1 $\beta$, IL-6, and TNF- $\alpha$, which were typically increased by irradiation. Apoptosis of the epithelial cells in the $\mathrm{Ndrg} 2^{\Delta \mathrm{G}}$ mice was significantly milder while the ratio of proliferation cells was larger in the epithelium of mice 8 days after TBI when compared with the WT mice.

Conclusions: These findings all indicated that NDRG2 deficiency in the intestine protects mice against radiation-induced GIS mainly through promoting proliferation and suppressing apoptosis of epithelial cells.

Keywords: N-Myc downstream regulated gene 2 (NDRG2); gastrointestinal syndrome (GIS); irradiation

Submitted Nov 03, 2020. Accepted for publication Jan 29, 2021.

doi: 10.21037/jgo-20-564

View this article at: http://dx.doi.org/10.21037/jgo-20-564

(c) Journal of Gastrointestinal Oncology. All rights reserved. 


\section{Introduction}

Acute radiation syndrome (ARS) is defined as an acute sickness that occurs when patients receive therapeutic or unexpected exposure to a super-high dose ( $>0.7$ grays) of ionizing radiation in a short period of time (1). In humans, the sensitivity to radiation of each organ is significantly different, and various radiation syndromes appear at varying doses of irradiation. At irradiation doses lower than 8 gray, hematopoietic stem cells are prone to suffer severe injury, resulting in immunological weakening and hemorrhagic tendency (2). Patients exposed to irradiation suffer from infection, hemorrhage, and even death within 30 days (hematopoietic syndrome; HPS) (3). At doses of more than 10 gray, villous epithelial cells and crypt stem cells, which are essential for the regeneration of colon villi and constitute the epithelial integrity $(4,5)$, were found to be growth-inhibited and even killed, causing epithelial damage, loss of intestinal barrier function, inflammation, and even gut-derived sepsis. Severe injury of gastrointestinal tract causes bacterial enteritis, malabsorption, diarrhea, and fluid loss, and has subacute lethality (gastrointestinal syndrome; GIS) $(4,6,7)$.

Supportive care, infection control, and a bone marrow transplant are performed as medical countermeasures against HPS, and can prevent death. However, there are currently no effective medical treatments for GIS, which greatly limits the clinical usage in abdominal radiotherapy $(4,6,7)$. Abundant evidence indicates that death of the epithelial stem cells in the crypts leads to GIS $(4,7)$, and it is thus particularly important to identify those novel genes which are crucial to the cell proliferation and cell death of villous epithelial cells and crypt stem cells.

$\mathrm{N}-\mathrm{Myc}$ downstream regulated gene 2 (NDRG2) was first cloned in our laboratory (8) and belongs to the NDRG family, which is characterized by an $\alpha / \beta$ hydrolase-fold motif and an esterase/lipase/thioesterase active site serine (9-11). $N D R G 2$ is a tumor-suppressor gene, and is associated with tumorigenesis, development, progression, and metastasis. Many kinds of tumors have been reported to have close associations with $N D R G 2$, including gastrointestinal tumors (12-15), breast cancer tumors (16-18), lung cancer tumors $(19,20)$, neurologic tumors (21-23) etc. In the tumor tissues, the expression level of $N D R G 2$ has been found to be considerably lower when compared with the level in paracarcinoma tissues of normal study groups. NDRG2 expression levels have been reported to be negatively correlated with TNM stage and lymph node metastasis.
Furthermore, a tend toward a decrease in NDRG2 expression levels with an advanced tumor grade and increased invasive depth of tumors has been observed (17,24-26).

In the GIS, villous epithelial cells and crypt stem cells are growth-inhibited and even killed, and epithelial damage, loss of intestinal barrier function, inflammation, and even gut-derived sepsis ensue. NDRG2 suppresses cell proliferation and promotes apoptosis, indicating that $N D R G 2$ may have a role in the progression of GIS. To analyze the role of $N D R G 2$ in GIS, we generated $\mathrm{Ndrg} 2^{\triangle \mathrm{G}}$ mice that lacked $N D R G 2$ specifically in the intestinal epithelium by crossing C57BL/6 mice which carry the floxed NDRG2 gene $\left(\mathrm{Ndrg} 2^{\mathrm{A} / \mathrm{H}}\right)$ with $\mathrm{C} 57 \mathrm{BL} / 6$ mice which carry the Cre recombinase and villin promoter (Vil/Cre).

Taken together, we found that $\mathrm{Ndrg} 2^{\Delta \mathrm{G}}$ mice showed significantly milder symptoms of GIS after 8 gray of total body irradiations (TBI), owing to reduction of cell death induced by irradiation and promotion of proliferation of crypt cells. Our findings provide a novel view into understanding the pathogenesis of GIS, and suggest that blockade of NDRG2 might be a novel target for mitigating intestinal radiation injury.

We present the following article in accordance with the ARRIVE reporting checklist (available at http://dx.doi. org/10.21037/jgo-20-564).

\section{Methods}

\section{Animal experiments}

All animal experiments were approved by the Animal Experiment Administration Committee of the Fourth Military Medical University. Experiments were performed in compliance with institutional guidelines for the care and use of animals. $\mathrm{Ndrg} 2^{\triangle \mathrm{G}}$ mice, lacking $N D R G 2$ specifically in the intestinal epithelium were first generated by hybridizing C57BL/6 mice possessing the floxed NDRG2 gene (Ndrg2fl/fl) with C57BL/6 mice carrying the Cre recombinase and villin promoter (Vil/Cre) (2). Mice were raised in cages in a specific pathogen-free (SPF) facility. Female $\mathrm{Ndrg} 2^{\Delta \mathrm{G}}$ mice aged $8-9$ weeks and with normal body weight (18.5-21.5 g) were adopted in the experiments. Wild-type (WT) female C57BL/6 mice, age-matched with $\mathrm{Ndrg} 2^{\Delta \mathrm{G}}$ mice, were used as the control group.

\section{Irradiation}

We used a ${ }^{60}$ Go source to irradiate the mice $(n=16$ per 
Table 1 Histopathological grading standard

\begin{tabular}{lllll}
\hline Scores & Epithelial deletion $(\%)$ & Crypt damage $(\%)$ & Goblet cell damage & Inflammatory cell infiltration \\
\hline 0 & Nonexistent & Nonexistent & Nonexistent & Nonexistent \\
1 & $0-5$ (mild) & $0-10($ mild $)$ & Mild & Mild \\
2 & $5-10$ (medium) & $10-20$ (medium) & Medium & Medium \\
3 & $>10$ (severe) & $>20($ severe) & Severe & Severe \\
\hline
\end{tabular}

Table 2 Primers for qRT-PCR in this study

\begin{tabular}{ll}
\hline Gene & Primer sequence \\
\hline IL-1 $\beta$ & Forward 5'-TAGACAACTGCACTACAGGCTCCGA-3' \\
& Reverse 5'-GGGTCCGACAGCACGAGGCT-3' \\
IL-6 & Forward 5'-CTGCAAGAGACTTCCATCCAG-3' \\
& Reverse 5'-AGTGGTATAGACAGGTCTGTTGG-3' \\
TNF- $\alpha$ & Forward 5'-ATGAGCACAGAAAGCATGATC-3' \\
& Reverse 5' -TACAGGCTTGTCACTCGAATT-3' \\
IL-10 & Forward 5'-TCAAGGCGCATGTGAACTCC-3' \\
& Reverse 5'-GATGTCAAACTCACTCATGGCT-3' \\
$\beta$-actin & Forward 5' -TGCGTGACATCAAAGAGAAG-3' \\
& Reverse 5' -TCCATACCCAAGAAGGAAGG-3' \\
\hline
\end{tabular}

group) to a lethal whole-body $\gamma$ radiation dose at 8 grays (dose rate $0.7 \mathrm{gray} / \mathrm{min}$ ). Then, we returned these mice to individual cages and monitored them carefully. We recorded the survival time of each mouse in the two groups and detected the degree of diarrhea and the loss of body weight to evaluate the severity of GIS.

\section{Assessment of bistopathological score}

We placed sections of jejunum in 5\% paraformaldehyde and stained them with hematoxylin and eosin (HE) according to manufacturer's instructions. Histopathological score represents the severity degree of irradiation injury to the small intestine, and the main indices of the assessment system in this study included epithelial deletion, crypt damage, goblet cell damage, and inflammatory cell infiltration. The detailed assessment used in this study is shown in Table 1.

\section{Myeloperoxidase (MPO) activity}

Jejunal myeloperoxidase (MPO) activity was considered as an index of neutrophil infiltration into the injured/ inflamed mucosa. We used an MPO biochemical kit (Service bio Technology Co. Ltd, Wuhan, China) to detect the level of MPO activity of the jejunal epithelium. The intestinal epithelium was isolated using procedures described previously (27). Concentrations of MPO in the jejunum are expressed as U/g. Every experiment was repeated three times.

\section{Enzyme-linked immunosorbent assay (ELISA) and reverse transcription-polymerase chain reaction (RT-PCR)}

For analysis of cytokines in epithelial cells, we used ELISA kits, including interleukin (IL)-6, IL-10, tumor necrosis factor alpha (TNF- $\alpha$ ); (Thermo Fisher Scientific, USA) to determine the protein levels of cytokines in the jejunum. For experiments of quantitative (RT-PCR), total RNA was extracted from the jejunal epithelium using TRIzol reagent (Invitrogen, USA). Total RNA (500 ng) was reverse transcribed to complementary DNA (cDNA) using SYBR Green PCR Light Cycler H, and real-time PCR was performed by Applied Biosystems PRISM 7500 Real-time PCR system (Thermo Fisher Scientific, USA). The detailed primer sequences are shown in Table 2 .

\section{Gene set enrichment analysis (GSEA) and differential analysis}

To validate the differential expression of NDRG2 between tumor and adjacent normal tissues, online analysis (https:// cistrome.shinyapps.io/timer/) with the key input term being "NDRG2" was performed. Moreover, to explore the biological function of NDRG2, GSEA was conducted, and the interested gene sets were Kyoto Encyclopedia of Genes and Genomes (KEGG) apoptosis and Reactome apoptosis pathway both downloaded from the Molecular Signatures Database (MSigDB, https://www.gsea-msigdb.org/gsea/ msigdb/index.jsp). In addition, there is no suitable RNA 
expression of colon after radiation. Thus, colorectal cancer (CRC) patients from TCGA database were selected to validate the potential function of NDRG2 by GSEA.

\section{Histology and immunobistochemistry}

We cut paraffin sections of jejunum $5 \mu \mathrm{m}$ size from the $\mathrm{Ndrg} 2^{\Delta \mathrm{G}}$ and WT mice and stained them with HE. Then, the height of the intestinal villus and crypts were measured by ImageJ software, and the histopathological scores of the samples were assessed under a light microscope. In the immunohistochemistry array, we conducted the similar procedure to my previous researches (2). Immunohistochemistry images were scanned by Panoramic MIDI (Santa Clara, CA, USA), and adjusted with a histochemical score (H-Score) by Quant Center.

\section{Statistical analysis}

Unpaired Student's $t$-test was used to compare the two mean values and one-way ANOVA analysis with Tukey's multiple comparison test adopted to compare three or more groups. Survival analysis was revealed by Kaplan-Meier curves and log-rank test was used as the main test of survival analysis. P value $<0.05$ in all data was considered statistically significant.

\section{Results}

\section{$\mathrm{Ndrg} 2^{\Delta G}$ mice were protected from intestinal radiation injury}

To explore the potential function of $N D R G 2$ in GIS, $\mathrm{Ndrg} 2^{\triangle \mathrm{G}}$ mice who lacked $N D R G 2$ in the intestinal epithelium were generated using the same procedure as that of a previous study (2). We recorded and analyzed the survival time of $\mathrm{Ndrg}^{\Delta \mathrm{G}}$ and WT mice after 8 Gray of TBI. Compared with the WT mice, $\mathrm{Ndrg}^{\Delta \mathrm{G}}$ mice showed longer survival, with a median survival time of 12.3 days, while the median survival time of WT mice was 9.5 days.

Kaplan-Meier curves showed longer survival time in $\mathrm{Ndrg} 2^{\Delta \mathrm{G}}$ mice than in WT mice after 8 Gray TBI: the median survival time of $\mathrm{Ndrg} 2^{\Delta \mathrm{G}}$ mice was 12.3 days and that of WT mice was 9.5 days (Figure $1 A, B$ ). The daily alternation of body weight showed that $\mathrm{Ndrg} 2^{\Delta \mathrm{G}}$ mice had milder weight loss compared with WT mice in days 1-5 (Figure 1C,D). However, weight loss showed no significant difference in days $6-10$, as $\mathrm{Ndrg}^{2 \mathrm{GG}}$ and $\mathrm{WT}$ mice both began to die of the acute injury on the sixth day after irradiation. Moreover, the results of the daily recording of diarrhea showed that $\mathrm{Ndrg} 2^{\Delta \mathrm{G}}$ mice had milder diarrhea compared with WT mice (Figure 1E). These results suggested that $\mathrm{Ndrg} 2^{\triangle \mathrm{G}}$ mice had significantly milder symptoms of radiation-induced GIS following TBI compared with WT mice.

\section{Deficiency of NDRG2 in the epithelium protected the jejunum from intestinal radiation injury}

To investigate whether the deficiency of NDRG2 in the mouse epithelium has a protective effect on intestinal radiation injury, we separated the whole gut from the duodenum to the anus 0,6 , and 8 days after 8 gray TBI. Compared to the WT mice, the jejunum of $\mathrm{Ndrg}^{\Delta \mathrm{G}}$ mice showed milder hemorrhage and edema (Figure $2 A$ ). Jejunal tissues were removed from the same position of the intestine, about $3 \mathrm{~cm}$ below the gastroduodenal junction and stained with HE on days 0 and 8 . We then measured the height of intestinal villus and crypts under a light microscope, and both $\mathrm{Ndrg} 2^{\Delta \mathrm{G}}$ and WT mice showed a reduced height of villus and crypts after TBI; however, the reduction of height in $\mathrm{Ndrg} 2^{\Delta \mathrm{G}}$ mice was less than that of WT mice, showing an improvement of intestine integrity (Figure 2B,C,D). Moreover, compared with WT mice, $\mathrm{Ndrg} 2^{\triangle \mathrm{G}}$ mice manifested lower histopathological scores and showed a lesser extent of epithelium deletion, crypt damage, goblet damage, and inflammatory cell infiltration (Figure 2E).

\section{Detecting the activity of MPO in the WT and $\mathrm{Ndrg} 2^{\Delta G}$ groups after irradiation}

MPO is a well-known parameter of neutrophil aggregation. Its activity was detected on day 8 after about 6 sessions of TBI. The results showed that MPO activity was decreased in the jejunal epithelium of $\mathrm{Ndrg}^{\triangle \mathrm{G}}$ mice compared to $\mathrm{WT}$ mice (Figure 2F).

\section{Detecting protein and $m R N A$ level of pro-inflammation cytokines of jejunal epithelial cells}

High levels of pro-inflammatory cytokines including IL-1 $\beta$, IL-6, and TNF- $\alpha$ can contribute to severe damage to jejunal tissue and barrier functions (2). To analyze the cytokines in jejunal epithelial cells, we detected the protein levels of cytokines in the jejunum using an ELISA kit and identified the mRNA levels using relative qRT-PCR. Consistent with decreased inflammatory score in the jejunum, $\mathrm{Ndrg} 2^{\Delta \mathrm{G}}$ mice 
A

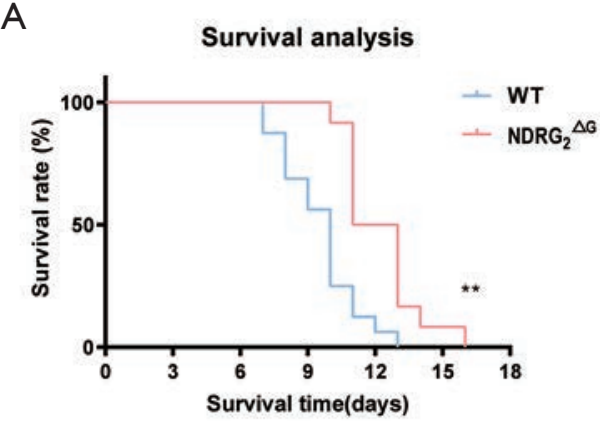

C

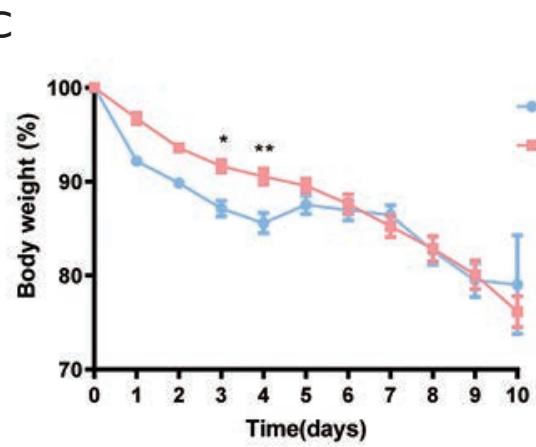

B

Survival time

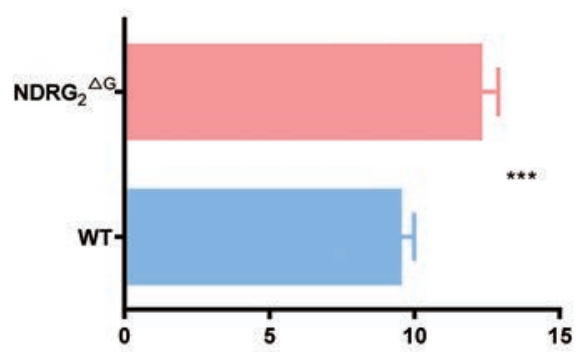

D

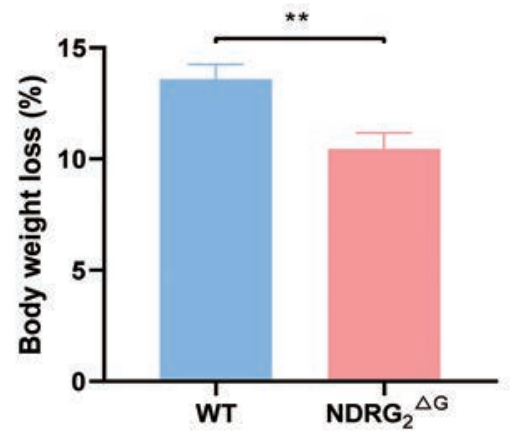

$E$

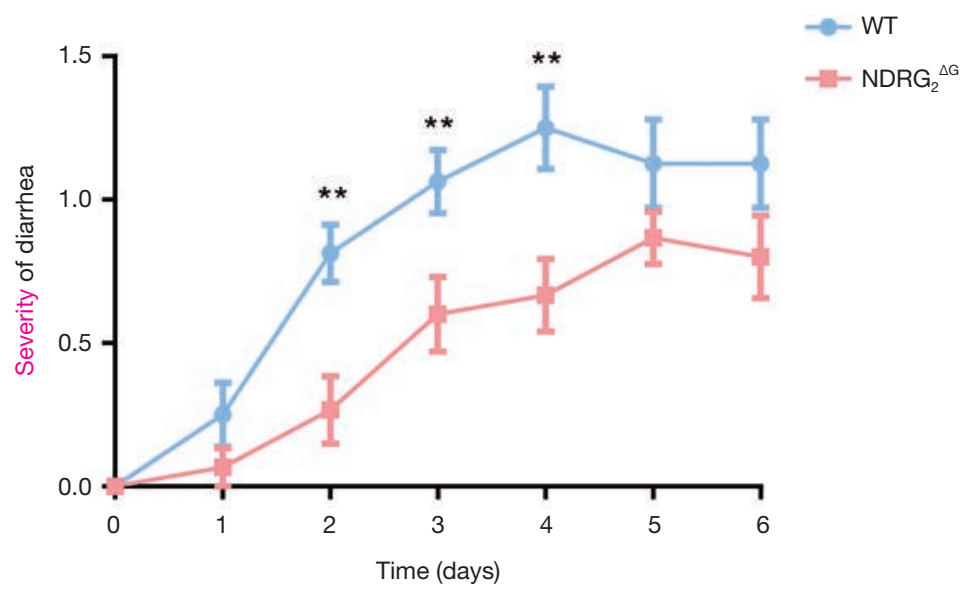

Figure 1 NDRG2 contributed to significantly severe symptoms of GIS. (A) Survival analysis of $\mathrm{Ndrg}^{\Delta \mathrm{G}}(\mathrm{n}=16)$ and WT mice $(\mathrm{n}=16)$ after 8 grays of TBI $\left({ }^{* *} \log\right.$-rank test, $\left.\mathrm{P}<0.01\right)$. (B) Average survival time of $\mathrm{Ndrg}_{2} 2^{\triangle \mathrm{G}}(\mathrm{n}=16)$ and $\mathrm{WT}$ group mice $(\mathrm{n}=16)$ after 8 grays of TBI $\left({ }^{* * *}\right.$ Wilcoxon test; $\left.\mathrm{P}<0.001\right)$. (C) $\mathrm{Ndrg} 2^{\triangle \mathrm{G}}$ mice had milder radiation-induced weight loss compared with WT mice on days $1-10$ after TBI; ${ }^{*}, \mathrm{P}<0.05$; ${ }^{* *}, \mathrm{P}<0.01$. (D) Mean body weight loss of $\mathrm{Ndrg} 2^{\Delta \mathrm{G}}$ mice and WT mice on day 5 after TBI; ${ }^{* *}, \mathrm{P}<0.01$. (E) $\mathrm{Ndrg} 2^{\Delta \mathrm{G}}$ mice showed milder diarrhea than WT mice; **, $\mathrm{P}<0.01$. GIS, radiation-induced gastrointestinal syndrome; WT, wildtype; TBI, total body $r$-irradiation.

showed significantly downregulated expression for IL-1 $\beta$, IL-6, and TNF- $\alpha$ (Figure $3 A, B, C, D, E, F$ ) and upregulated expression for IL-10 (Figure $3 G, H$ ). In conclusion, $\mathrm{Ndrg} 2^{\Delta \mathrm{G}}$ mice showed decreased jejunal cytokine expression in both mRNA and protein level compared with WT mice after TBI.

\section{Potential signaling patbway in the high NDRG2 group}

By comparing the 21 kinds of tumor and normal samples, we found that the expression of NDRG2 was significantly downregulated in 17 kinds of cancerous tissues compared to normal tissues (Figure 4A). Thereafter, GSEA analysis 
A

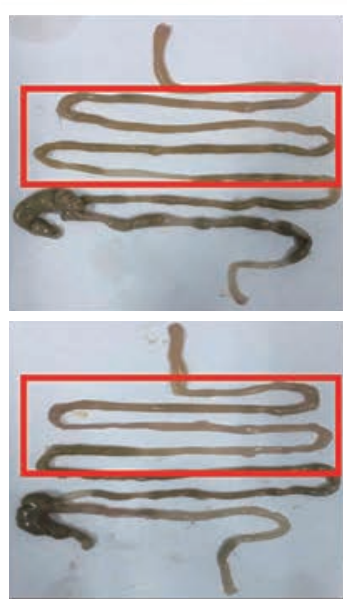

B

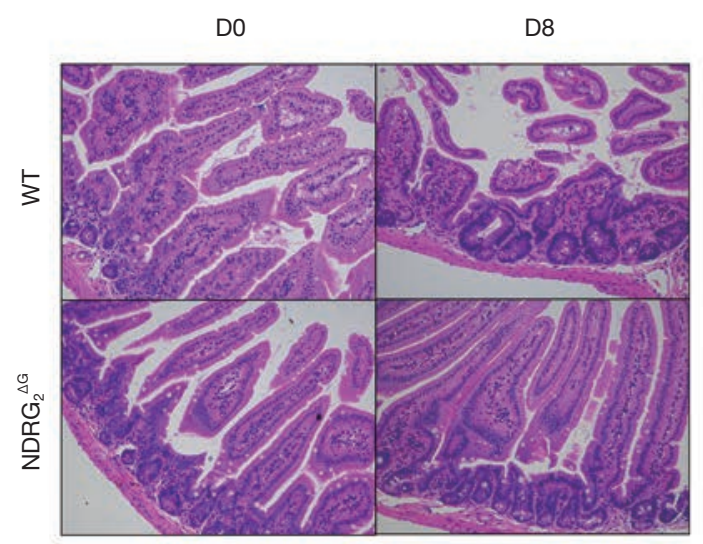

$E$

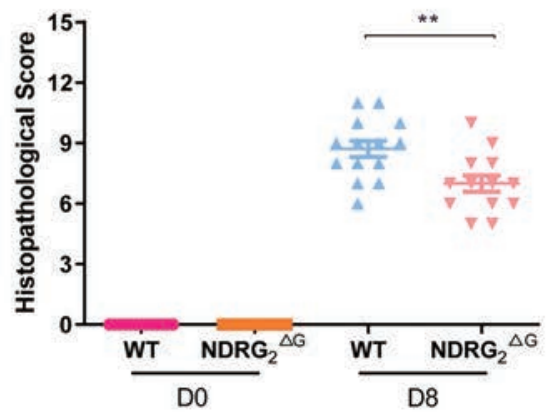

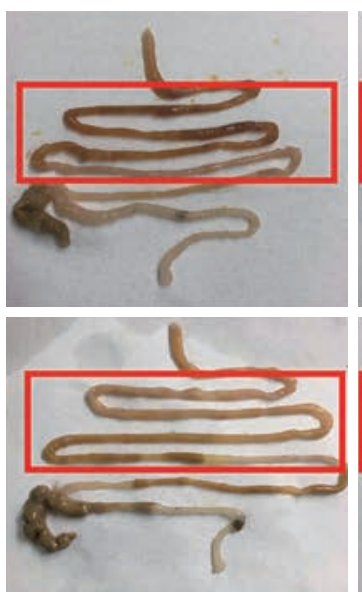
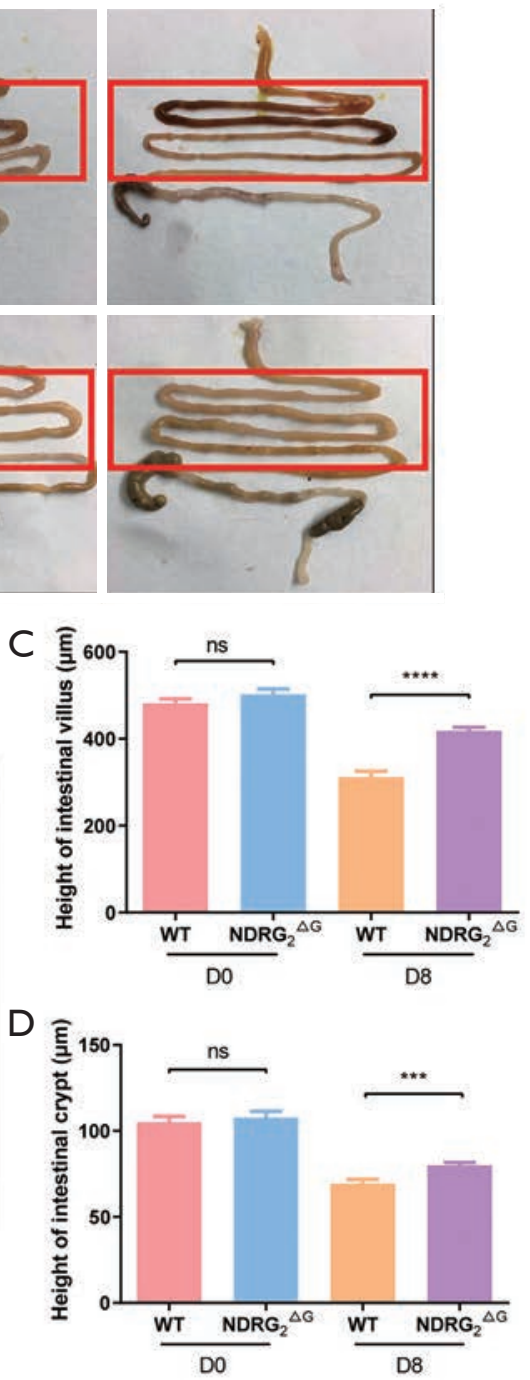

$\mathrm{F}$

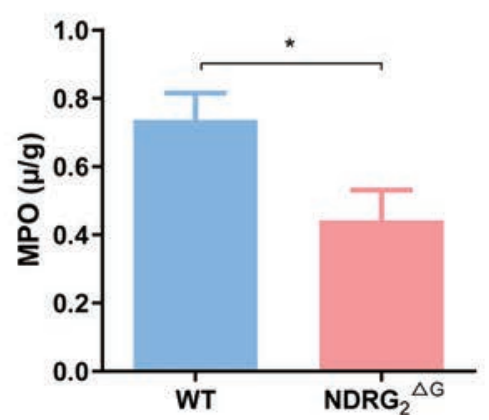

Figure 2 Deletion of NDRG2 in the mice epithelium protected the jejunum from intestinal radiation injury and reduced MPO activity. (A) Representative intestinal images from the $\mathrm{Ndrg}^{\triangle \mathrm{G}}$ and $\mathrm{WT}$ groups on day 0,6 , and 8 after TBI. (B) Representative HE-stained jejunal sections (magnification, 20×). (C) Height of the intestinal villus (ns, no significance; ${ }^{* * * *}, \mathrm{P}<0.0001$ ) and (D) intestinal crypt $\left.{ }^{* * *}, \mathrm{P}<0.001\right)$. (E) Histology scores of the $\mathrm{Ndrg}^{\triangle \mathrm{G}}$ and $\mathrm{WT}$ groups on days 0 and 8 respectively; values are expressed as the mean $\pm \mathrm{SD}$; ${ }^{* *}, \mathrm{P}<0.01$. (F) MPO levels in the jejunal epithelium of the $\mathrm{Ndrg} 2^{\Delta \mathrm{G}}$ and $\mathrm{WT}$ groups on day 8 after TBI; * $\mathrm{P}<0.05$. MPO, myeloperoxidase; TBI, total body $\gamma$-irradiation; HE-stained, hematoxylin and eosin stained; WT, wildtype; SD, standard deviation. 
A

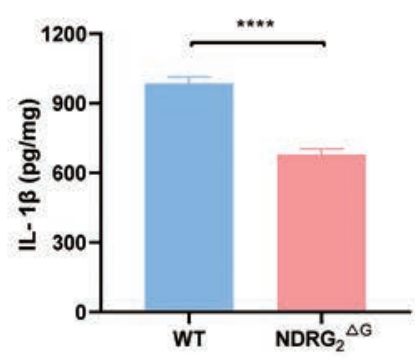

B

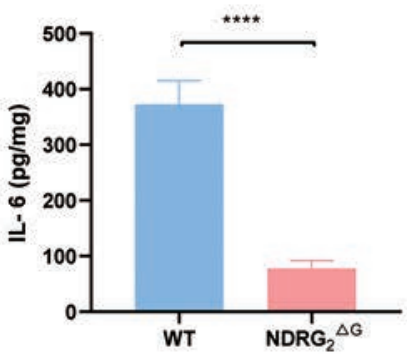

E

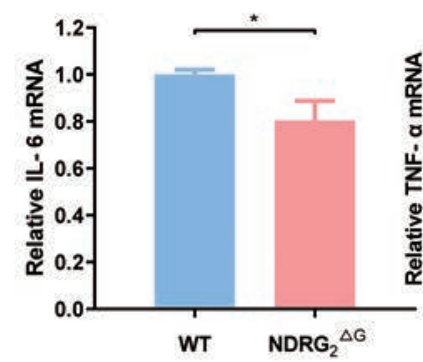

F

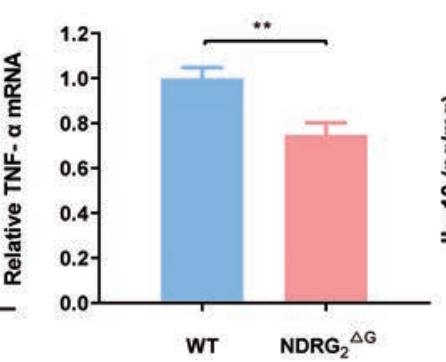

C

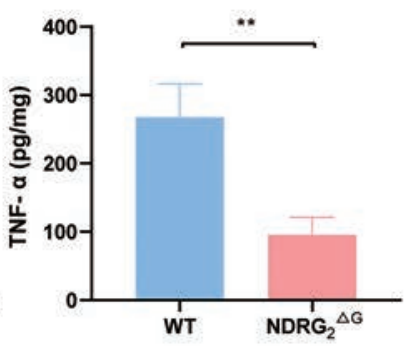

G

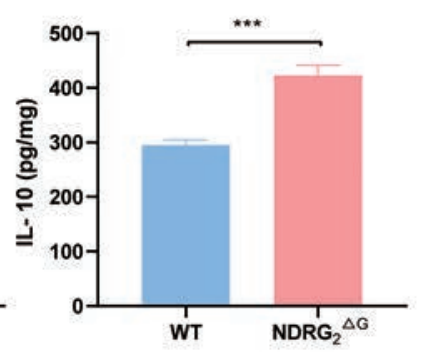

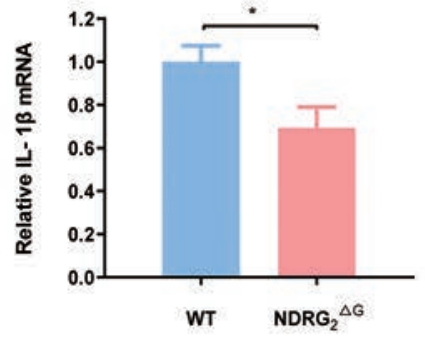

$\mathrm{H}$

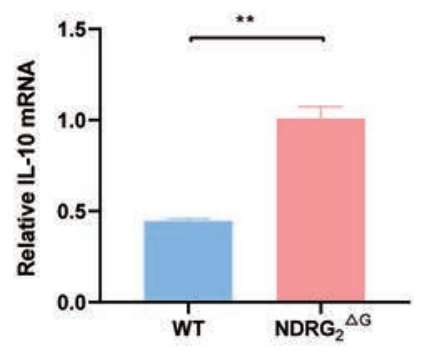

Figure 3 Proteins and mRNA levels of pro- and anti-inflammatory cytokines. (A,B,C) represent the proteins level of IL-1 $\beta$, IL-6, and TNF- $\alpha$ on day 8 after TBI. mRNA levels of IL-1 $\beta$ (D), IL-6 (E), and TNF- $\alpha$ (F) on day 8. Proteins (G) and mRNA (H) level of antiinflammatory cytokines IL-10 on day 8 after TBI. Unpaired Student's t-test was performed to validate the difference of WT and Ndrg $2^{\triangle \mathrm{G}}$ groups. *, $\mathrm{P}<0.05$; **, $\mathrm{P}<0.01$; ***, $\mathrm{P}<0.001$; ****, $\mathrm{P}<0.0001$. mRNA, messenger RNA; TBI, total body $\gamma$-irradiation; WT, wildtype.

was adopted to identify the relationship of NDRG2 with apoptosis, and results suggested the two similar apoptosis gene sets were commonly mapped in the high expression $N D R G 2$ group than in the low expression group with CRC (Figure $4 B, C$ ). Likewise, in western blotting assay, the protein of activated form caspase 3 (cleaved caspase 3) was decreased 8 days after TBI, with the $N d r g 2^{\Delta G}$ group having lower caspase 3 levels than the WT group (Figure 4D), indicating that NDRG2 depletion could ameliorate the radiation-induced GIS mainly through enhancing cell apoptosis after excessive radiation.

\section{Acute radiation-induced apoptosis and cell renewal in WT and $\mathrm{Ndrg} 2^{\Delta G}$ mice after TBI}

To further validate the role of NDRG2 in apoptosis, we measured epithelial cell apoptosis by counting TUNEL(Terminal deoxynucleotidyl transferase-mediated dUTP-biotin nick end labeling)-positive cells on day 8 after TBI. In Ndrg2 ${ }^{\Delta \mathrm{G}}$ mice, there were less TUNEL-positive cells than in WT mice after irradiation (Figure $5 A$ ). We also analyzed cell apoptosis with Bax and caspase 3 staining, and consistent with the results of TUNEL, we found that the number of apoptotic cells was lower in $\mathrm{Ndrg} 2^{\Delta \mathrm{G}}$ mice than in WT mice. Moreover, we analyzed cell anti-apoptosis with B-cell lymphoma 2 (Bcl-2) staining and, as expected, the proportion of anti-apoptotic cells in $\mathrm{Ndrg}^{2 \mathrm{GG}}$ mice was larger than that in WT mice. The results suggested that $N D R G 2$ deficiency in the intestine suppressed cell apoptosis and reduced the damage to the intestine villus and crypt (Figure 5B). To analyze cell proliferation, we quantitatively evaluated the cells with Ki-67 and proliferating cell nuclear antigen (PCNA) staining. Compared to WT mice, there was a higher rate of cell proliferation in $\mathrm{Ndrg} 2^{\Delta G}$ mice after TBI, which subsequently promoted the repair of jejunal tissue (Figure 5C,D).

\section{Discussion}

Accidental or therapeutic exposure to high-dose radiation results in acute radiation syndrome, which includes two main life-threatening syndromes: HPS and GIS $(4,5)$. Death of villous epithelial cells and crypt stem cells is the leading cause of radiation-induced GIS. However, 


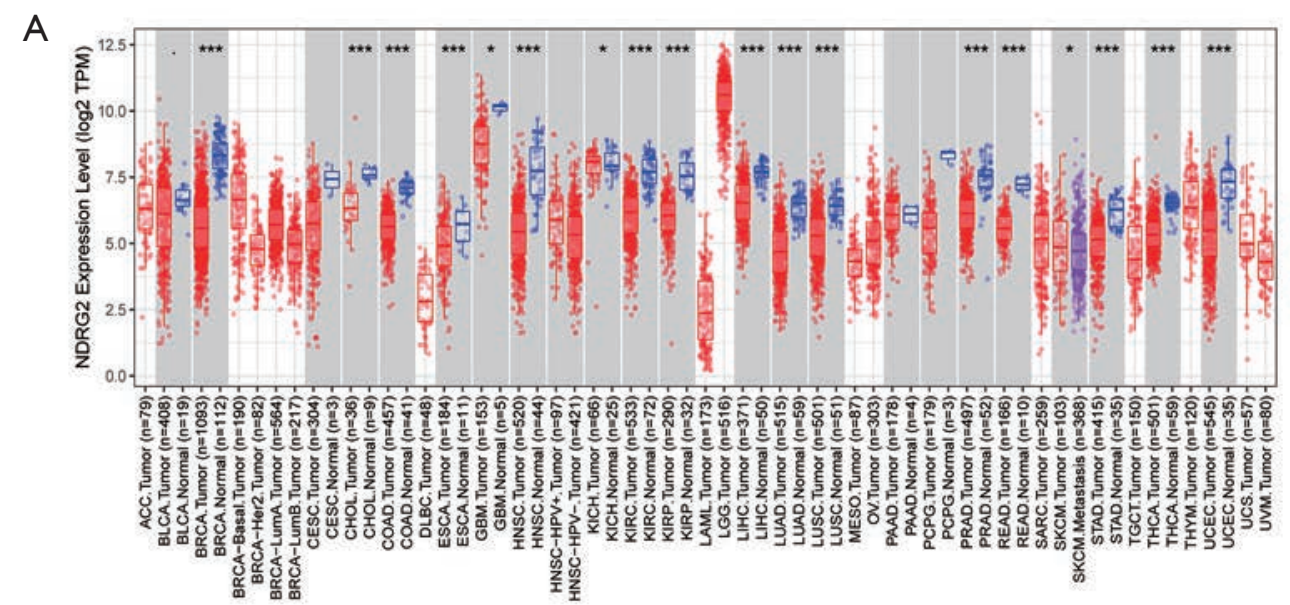

B

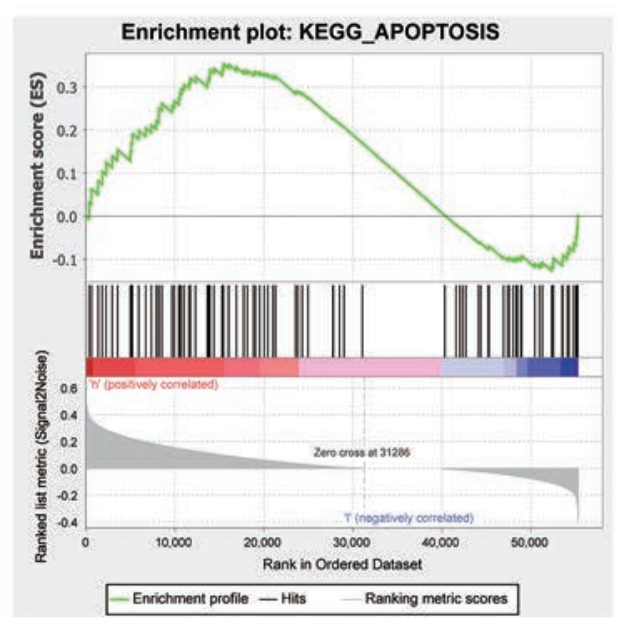

C
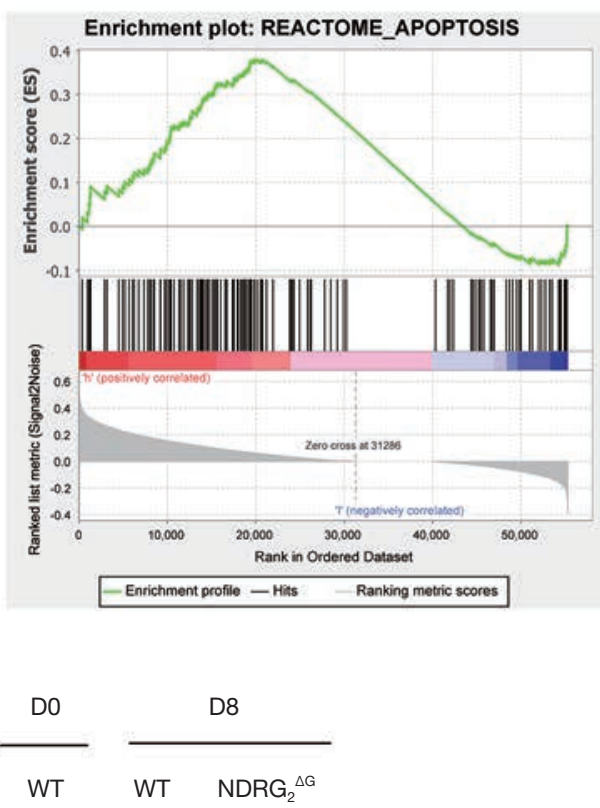

cleaved caspase-3

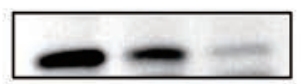

NDRG2

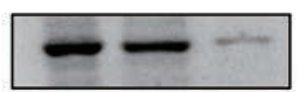

Actin

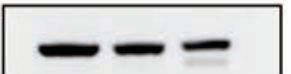

Figure 4 Relationship between NDRG2 and apoptosis. Different mRNA expressions of NDRG2 in 22 types of tumor and their corresponding adjacent samples. (A) Red boxes represent cancerous tissues and blue boxes represent tumor tissues. GSEA analysis was revealed in high- and low-expression NDRG2 groups in CRC. Two apoptosis-related gene sets, KEGG apoptosis (B) and Reactome apoptosis (C) were mapped respectively. (D) Western blotting showed the alternation of apoptosis-related genes in WT and Ndrg2 ${ }^{\Delta \mathrm{G}}$ mice after days 0 and $8 .{ }^{*}, \mathrm{P}<0.05 ;{ }^{* * *}, \mathrm{P}<0.001$. GSEA, Gene set enrichment analysis; CRC, colorectal cancer; KEGG, Kyoto encyclopedia of genes and genomes; WT, wildtype. 
A
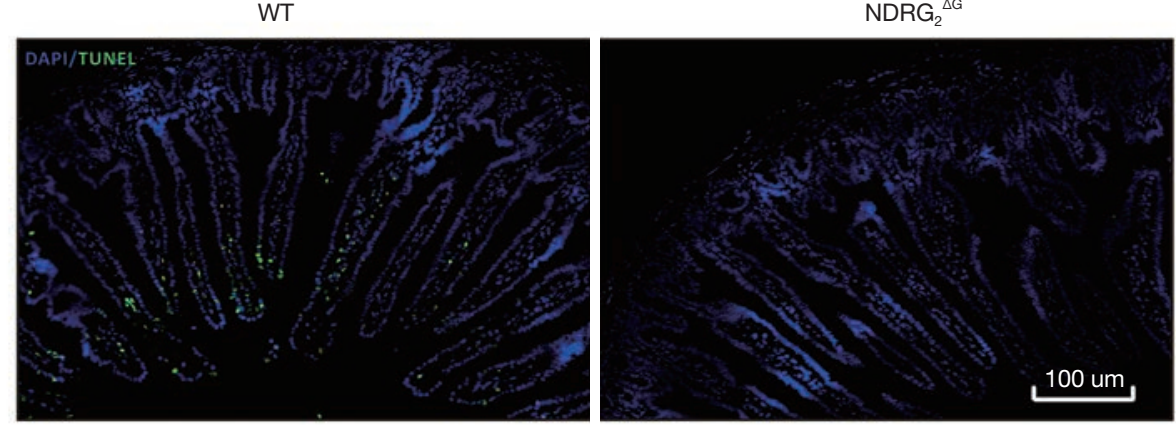

B
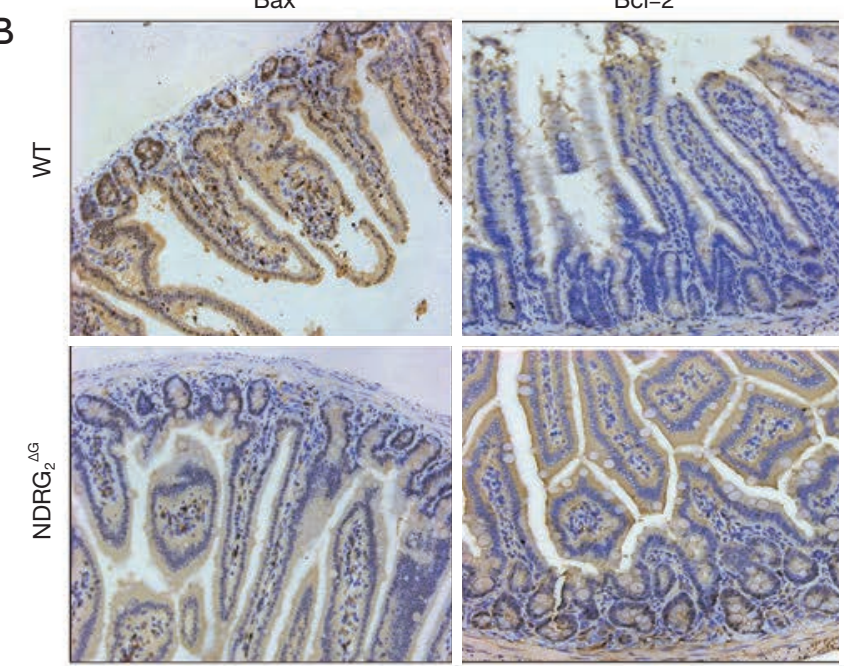

C
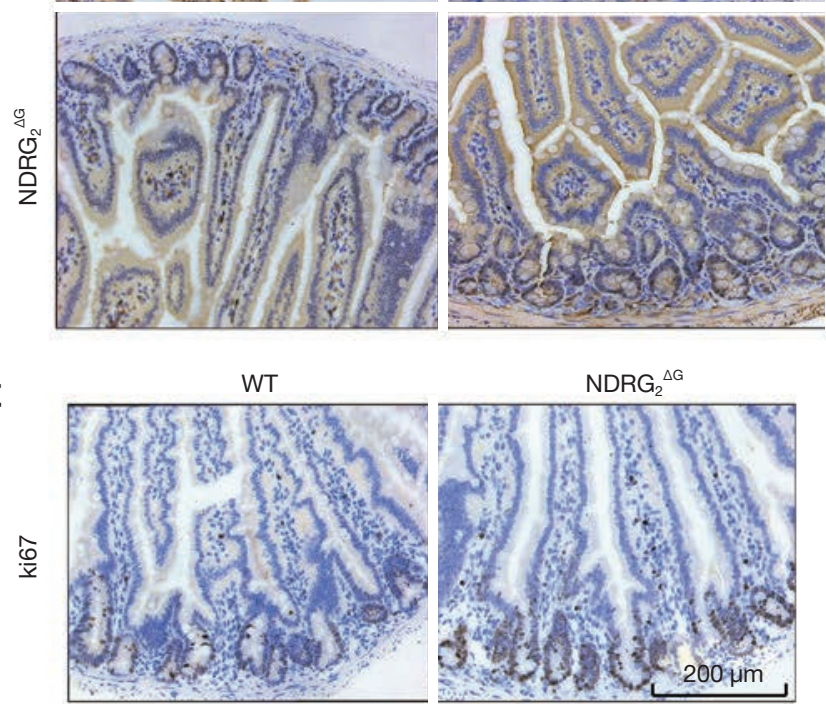

D

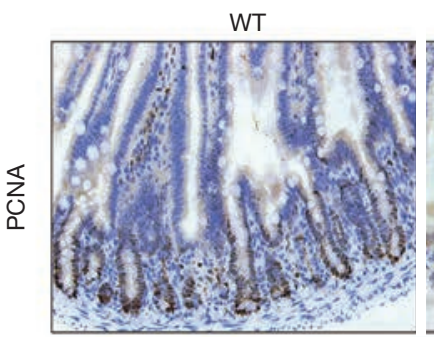

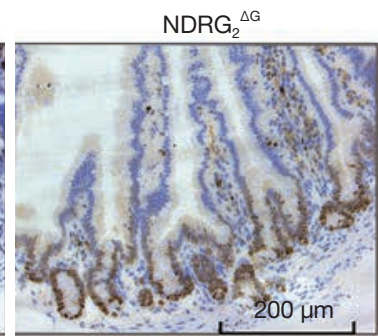

caspase-3
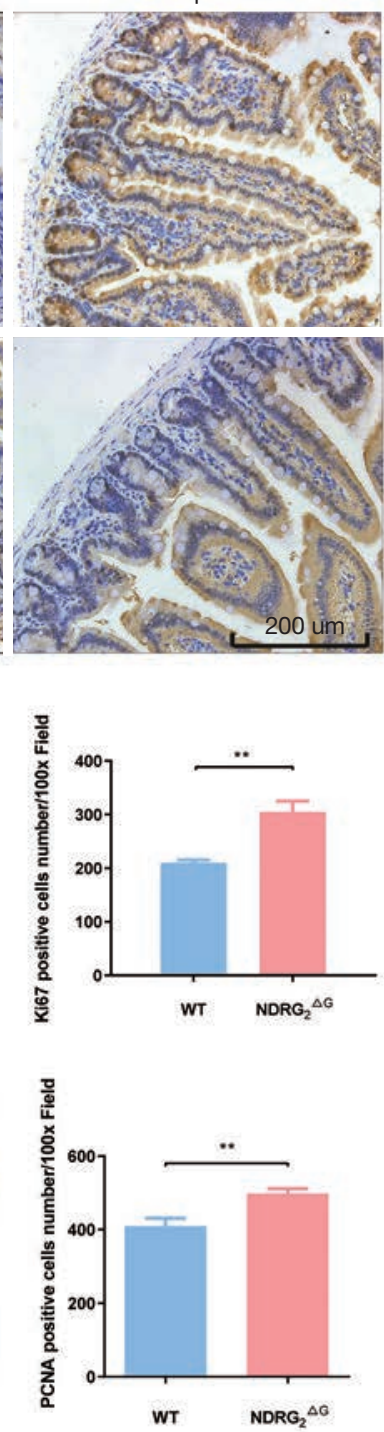

Figure 5 Influence of NDRG2 deficiency in cell apoptosis of the epithelium and cell proliferation of villus and crypt. (A) TUNEL-positive cells in Ndrg $2^{\Delta \mathrm{G}}$ mice and WT mice on day 8 after TBI. (B) Analysis of cell apoptosis with staining of Bax, caspase 3, and Bcl-2 (magnification, 50x). (C) Quantitative analysis of cell proliferation with Ki-67 and (D) PCNA staining (magnification, 50×). **, $\mathrm{P}<0.01$. WT, wildtype; TBI, total body $\gamma$-irradiation; TUNEL, terminal deoxynucleotidyl transferase-mediated dUTP-biotin nick end labeling; Ki-67, antigen KI67; PCNA, proliferating cell nuclear antigen. 
there exist no effective interventions or therapies that can mitigate intestinal damage or reduce the mortality of radiation-injured patients (28). There is thus an urgent need to discover those genes that are integral to regulating the cell proliferation and cell death of these epithelial and stem cells. In this study, NDRG2 deficiency in the intestine could protect mice against radiation-induced GIS and decrease the lethality induced by irradiation through promoting cell proliferation and reducing cell apoptosis of intestinal villus and crypts.

In our previous study, we identified the function of NDRG2 in apoptosis and cell proliferation in various cancer cells. Specifically, we found that the proliferation rate of A-498 clear cell renal cell carcinoma (CCRCC) cells significantly decreased when infected by NDRG2 recombinant adenovirus, and NDRG2 could induce CCRCC cycle arrested at the G1 phase (29). In addition, p53-mediated apoptosis was decreased by silencing of NDRG2 in the osteosarcoma cells and lung cancer cells (30). Likewise, the p53-induced apoptosis was enhanced by the overexpression of NDRG2 in the hepatocarcinoma cells (HepG2 and Huh7) (14). The apoptosis-promoting effect of NDRG2 was also discovered in CCRCC cells (30), esophageal squamous-cell carcinoma (ESCC) cells (31), and MKN28 cells (12). These findings revealed that NDRG2 was strongly involved in cell proliferation and apoptosis, indicating its potential role in radiation-induced GIS.

Although an increasing number of studies focusing on the function of NDRG2 in cell proliferation and apoptosis have been conducted in tumor cells, no research exists concerning its role in irradiation-induced GIS. To address this, we were first to generate $\mathrm{Ndrg} 2^{\Delta \mathrm{G}}$ mice that lacked $N D R G 2$ specifically in the intestinal epithelium. We found that after 8 gray of total body $r$-irradiation, the deletion of $N D R G 2$ in the intestine revealed considerably milder symptoms of GIS, including those of mortality (Figure 1A,B), body weight loss (Figure 1C,D), and severity of diarrhea (Figure 1C) with less extensive injury to jejunal tissue (Figure $2 A$ ), compared with the WT mice. Moreover, the $\mathrm{Ndrg} 2^{\Delta \mathrm{G}}$ mice significantly inhibited the messenger RNA (mRNA) (Figure 3D,E,F) and protein expression (Figure $3 A, B, C$ ) of pro-inflammatory IL-1 $\beta$, IL-6, and TNF- $\alpha$ which can exacerbate symptoms and contribute to the high morality caused by irradiation. Likewise, the level of anti-inflammatory IL-10 was higher in $\mathrm{Ndrg} 2^{\Delta \mathrm{G}}$ mice than WT mice (Figure $3 G, H$ ). Additionally, differential analysis (Figure $4 A$ ) indicated that NDRG2 had a marked decline in expression in most cancerous tissue compared to adjacent samples, suggesting that NDRG2 contributes to tumor suppression. It appears that $N D R G 2$ has a close correlation with p53-related apoptosis, and GSEA enrichment (Figure 4B,C) indicated the same conclusion: the high NDRG2 group showed a higher level of apoptosis than its low-expression counterpart. Regarding the alternation of apoptosis in $\mathrm{WT}$ and $\mathrm{Ndrg} 2^{\triangle \mathrm{G}}$ mice, we performed a series of assay including western blotting, TUNEL staining, histology, and immunohistochemistry. Compared with the WT group on day 8 , the $\mathrm{Ndrg} 2^{\Delta \mathrm{G}}$ group had less cleaved caspase 3 (Figure $4 D$ ), as revealed by western blotting. Similarly, fewer TUNEL-positive epithelial cells were present in Ndrg2 ${ }^{\Delta \mathrm{G}}$ than in WT mice on day 8 (Figure 5A). Immunohistochemistry assay showed that the two pro-apoptosis proteins (Bax and caspase 3) were downregulated in the $\mathrm{Ndrg}^{\Delta \mathrm{G}}$ group while the anti-apoptosis protein $(\mathrm{Bcl}-2)$ was upregulated in the WT mice on day 8 (Figure 5B). Ki-67 and PCNA were the two main molecules used to evaluate the cell proliferation, which showed that the number of proliferated cells was larger in the epithelium of mice 8 days after TBI exposure than in the WT mice. Collectively, the NDRG2-deficient mice had a better outcome and milder GIS. Mechanistically, NDRG2 (KO) could bring about the lower levels of pro-inflammatory cytokines, higher levels of anti-inflammation cytokines (IL-10), less cell apoptosis, and greater cell proliferation of intestinal villus and crypt after radiation.

In our study, we speculate that NDRG2 deficiency in the intestine plays a crucial role in suppressing cell apoptosis and promoting cell proliferation of intestinal villus and crypt, suggesting that NDRG2 might be a novel therapeutic target for alleviating irradiation-induced intestinal injury.

\section{Acknowledgments}

Funding: This work was financially supported by the National Natural Science Foundation of China (No. 31571437 and 81770523), the Key Research and Development Program of Shaanxi (No. 2019XC008 and 2019JC22), and the State Key Laboratory of Cancer Biology Project (No. CBSKL2019ZZ11).

\section{Footnote}

Reporting Checklist: The authors have completed the ARRIVE reporting checklist. Available at http://dx.doi. org/10.21037/jgo-20-564

Data Sharing Statement: Available at http://dx.doi. org/10.21037/jgo-20-564 
Conflicts of Interest: All authors have completed the ICMJE uniform disclosure form (available at http://dx.doi. org/10.21037/jgo-20-564). The authors have no conflicts of interest to declare.

Ethical Statement: The authors are accountable for all aspects of the work in ensuring that questions related to the accuracy or integrity of any part of the work are appropriately investigated and resolved. All animal experiments were approved by the Animal Experiment Administration Committee of the Fourth Military Medical University. Experiments were performed in compliance with institutional guidelines for the care and use of animals.

Open Access Statement: This is an Open Access article distributed in accordance with the Creative Commons Attribution-NonCommercial-NoDerivs 4.0 International License (CC BY-NC-ND 4.0), which permits the noncommercial replication and distribution of the article with the strict proviso that no changes or edits are made and the original work is properly cited (including links to both the formal publication through the relevant DOI and the license). See: https://creativecommons.org/licenses/by-nc-nd/4.0/.

\section{References}

1. Andreyev HJ, Wotherspoon A, Denham JW, et al. "Pelvic radiation disease": new understanding and new solutions for a new disease in the era of cancer survivorship. Scand J Gastroenterol 2011;46:389-97.

2. Li H, Liang Y, Lai X, et al. Genetic Deletion of Fbw7 in the mouse intestinal epithelium aggravated dextran sodium sulfate-induced colitis by modulating the inflammatory response of NF- $\kappa \mathrm{B}$ pathway. Biochem Biophys Res Commun 2018;498:869-76.

3. Dörr H, Meineke V. Acute radiation syndrome caused by accidental radiation exposure - therapeutic principles. BMC Med 2011;9:126.

4. Waselenko JK, MacVittie TJ, Blakely WF, et al. Medical management of the acute radiation syndrome: recommendations of the Strategic National Stockpile Radiation Working Group. Ann Intern Med 2004;140:1037-51.

5. Koenig KL, Goans RE, Hatchett RJ, et al. Medical treatment of radiological casualties: current concepts. Ann Emerg Med 2005;45:643-52.

6. Mettler FA Jr, Voelz GL. Major radiation exposure-what to expect and how to respond. N Engl J Med
2002;346:1554-61.

7. Berger ME, Christensen DM, Lowry PC, et al. Medical management of radiation injuries: current approaches. Occup Med (Lond) 2006;56:162-72.

8. Yang XL, Zhang YL, Yao LB, et al. Cloning, sequencing and bioinformatics analysis of a new tumor suppressor gene ndr2 from mouse. Ai Zheng 2003;22:230-4.

9. Melotte V, Qu X, Ongenaert M, et al. The N-myc downstream regulated gene (NDRG) family: diverse functions, multiple applications. FASEB J 2010;24:4153-66.

10. Qu X, Zhai Y, Wei H, et al. Characterization and expression of three novel differentiation-related genes belong to the human NDRG gene family. Mol Cell Biochem 2002;229:35-44.

11. Shaw E, McCue LA, Lawrence CE, et al. Identification of a novel class in the alpha/beta hydrolase fold superfamily: the $\mathrm{N}$-myc differentiation-related proteins. Proteins 2002;47:163-8.

12. Tao $Y$, Guo $Y$, Liu W, et al. AKT inhibitor suppresses hyperthermia-induced Ndrg2 phosphorylation in gastric cancer cells. Braz J Med Biol Res 2013;46:394-404.

13. Chu D, Zhang Z, Li Y, et al. Prediction of colorectal cancer relapse and prognosis by tissue mRNA levels of NDRG2. Mol Cancer Ther 2011;10:47-56.

14. Cao W, Zhang JL, Feng DY, et al. The effect of adenovirus-conjugated NDRG2 on p53-mediated apoptosis of hepatocarcinoma cells through attenuation of nucleotide excision repair capacity. Biomaterials 2014;35:993-1003.

15. Shen $\mathrm{L}, \mathrm{Qu} X, \mathrm{Li} H$, et al. NDRG2 facilitates colorectal cancer differentiation through the regulation of Skp2-p21/ p27 axis. Oncogene 2018;37:1759-74.

16. Ma J, Liu W, Guo H, et al. N-myc downstream-regulated gene 2 expression is associated with glucose transport and correlated with prognosis in breast carcinoma. Breast Cancer Res 2014;16:R27.

17. Lorentzen A, Lewinsky RH, Bornholdt J, et al. Expression profile of the N-myc Downstream Regulated Gene 2 (NDRG2) in human cancers with focus on breast cancer. BMC Cancer 2011;11:14.

18. Zheng J, Liu Q, Li Y, et al. NDRG2 expression regulates CD24 and metastatic potential of breast cancer cells. Asian Pac J Cancer Prev 2010;11:1817-21.

19. Wang H, Wang W, Wang X, et al. Reduced N-Myc downstream-regulated gene 2 expression is associated with CD24 upregulation and poor prognosis in patients with lung adenocarcinoma. Med Oncol 2012;29:3162-8.

20. Li R, Yu C, Jiang F, et al. Overexpression of N-Myc 
downstream-regulated gene 2 (NDRG2) regulates the proliferation and invasion of bladder cancer cells in vitro and in vivo. PLoS One 2013;8:e76689.

21. Zhang ZG, Li G, Feng DY, et al. Overexpression of NDRG2 can inhibit neuroblastoma cell proliferation through negative regulation by CYR61. Asian Pac J Cancer Prev 2014;15:239-44.

22. Li L, Wang J, Shen X, et al. Expression and prognostic value of NDRG2 in human astrocytomas. J Neurol Sci 2011;308:77-82.

23. Lusis EA, Watson MA, Chicoine MR, et al. Integrative genomic analysis identifies NDRG2 as a candidate tumor suppressor gene frequently inactivated in clinically aggressive meningioma. Cancer Res 2005;65:7121-6.

24. Park MY, Choi SC, Lee HS, et al. A quantitative analysis of N-myc downstream regulated gene 2 (NDRG 2) in human tissues and cell lysates by reverse-phase protein microarray. Clin Chim Acta 2008;387:84-9.

25. Piepoli A, Cotugno R, Merla G, et al. Promoter methylation correlates with reduced NDRG2 expression in advanced colon tumour. BMC Med Genomics 2009;2:11.

26. Feng L, Xie Y, Zhang H, et al. Down-regulation of
NDRG2 gene expression in human colorectal cancer involves promoter methylation and microRNA-650. Biochem Biophys Res Commun 2011;406:534-8.

27. Satoh Y, Williams MR, Habara Y. Effects of AIF4and ATP on intracellular calcium dynamics of crypt epithelial cells in mouse small intestine. Cell Tissue Res 1999;298:295-305.

28. Romesser PB, Kim AS, Jeong J, et al. Preclinical murine platform to evaluate therapeutic countermeasures against radiation-induced gastrointestinal syndrome. Proc Natl Acad Sci U S A 2019;116:20672-8.

29. Ma JJ, Liao CG, Jiang X, et al. NDRG2 suppresses the proliferation of clear cell renal cell carcinoma cell A-498. J Exp Clin Cancer Res 2010;29:103.

30. Liu N, Wang L, Li X, et al. N-Myc downstream-regulated gene 2 is involved in p53-mediated apoptosis. Nucleic Acids Res 2008;36:5335-49.

31. Shi H, Li N, Li S, et al. Expression of NDRG2 in esophageal squamous cell carcinoma. Cancer Sci 2010;101:1292-9.

(English Language Editor: J. Gray)
Cite this article as: Zhu J, Tian L, Li H, Hao J, Wang S, Li J, Zhang J. Radiation-induced gastrointestinal syndrome is alleviated in NDRG2-deficient mice. J Gastrointest Oncol 2021;12(1):100-111. doi: 10.21037/jgo-20-564 